\title{
Spectral Characterizations of Some Distance-Regular Graphs
}

\author{
EDWIN R. VAN DAM \\ Edwin.vanDam@kub.nl \\ WILLEM H. HAEMERS \\ Haemers@kub.nl \\ Tilburg University, Department of Econometrics and O.R., PO Box 90153, 5000 LE Tilburg, The Netherlands
}

Received August 14, 2000; Revised July 31, 2001

Abstract. When can one see from the spectrum of a graph whether it is distance-regular or not? We give some new results for when this is the case. As a consequence we find (among others) that the following distance-regular graphs are uniquely determined by their spectrum: The collinearity graphs of the generalized octagons of order $(2,1),(3,1)$ and $(4,1)$, the Biggs-Smith graph, the $M_{22}$ graph, and the coset graphs of the doubly truncated binary Golay code and the extended ternary Golay code.

Keywords: distance regular graphs, eigenvalues, cospectral graphs

\section{Introduction}

Suppose $G$ is a distance-regular graph with diameter $d$ and intersection array

$$
\Upsilon=\left\{b_{0}, \ldots, b_{d-1} ; c_{1}, \ldots, c_{d}\right\} .
$$

A central question in the theory of distance-regular graphs is: Does $\Upsilon$ determine $G$ ? For many distance-regular graphs the answer is affirmative, for many the answer is negative, and in many cases the answer is still undecided (see [1]). Let

$$
\Sigma=\left\{\left[\lambda_{0}\right]^{1},\left[\lambda_{1}\right]^{m_{1}}, \ldots,\left[\lambda_{d}\right]^{m_{d}}\right\}
$$

be the spectrum of $G$, that is, the multiset of eigenvalues of the adjacency matrix of $G$ (exponents denote multiplicities), with $\lambda_{0}$ as largest eigenvalue. It is well known that $\Upsilon$ determines $\Sigma$. So it seems natural to ask the more restrictive question: Is $G$ determined by $\Sigma$ ? If it is known that $G$ is distance-regular, then $\Sigma$ also determines $\Upsilon$ (see Lemma 4 in the appendix), hence in this case the two questions are equivalent. Thus the problem that needs to be solved is: Is a graph $G^{\prime}$ with the same spectrum $\Sigma$ as $G$ necessarily distance-regular? This is in general not the case. There exist (many) counter examples for all $d \geq 3$ (see [6]). However, the answer is known to be affirmative in several special cases such as: If $d \leq 2$, if $G$ is a generalized Odd graph (see [9]), if $d=3$ and $c_{2}=1$ (see [6]), or if the girth $g$ satisfies $g \geq 2 d-1$ (see [2,6]) (note that the first condition is a special case of the last one).

In the present paper we find a few more such sufficient conditions. Examples of graphs satifying these new conditions are given in Tables 1 and 2; indeed Table 1 gives those 
Table 1. Distance-regular graphs uniquely determined by their spectra.

\begin{tabular}{|c|c|c|c|}
\hline Graph & Spectrum & Intersection array & Ref. \\
\hline $\begin{array}{l}\text { Collinearity graph } \\
\text { gen. octagon }(s, 1) \\
s=2,3,4\end{array}$ & $\begin{array}{l}\left\{[2 s]^{1},[s-1+\sqrt{2 s}]^{\frac{1}{2} s(s+1)^{2}},[s-1]^{s\left(s^{2}+1\right)}\right. \\
\left.\quad[s-1-\sqrt{2 s}]^{\frac{1}{2} s(s+1)^{2}},[-2]^{s^{4}}\right\}\end{array}$ & $\{2 s, s, s, s ; 1,1,1,2\}$ & Corol. 4 \\
\hline $\begin{array}{l}\text { Collinearity graph } \\
\text { gen. dodecagon }(2,1)\end{array}$ & $\begin{array}{l}\left\{[4]^{1},[1+\sqrt{6}]^{21},[1+\sqrt{2}]^{27},[1]^{28}\right. \\
\left.\quad[1-\sqrt{2}]^{27},[1-\sqrt{6}]^{21},[-2]^{64}\right\}\end{array}$ & $\begin{array}{l}\{4,2,2,2,2,2 \\
\quad 1,1,1,1,1,2\}\end{array}$ & Corol. 4 \\
\hline$M_{22}$ graph & $\left\{[7]^{1},[4]^{55},[1]^{154},[-3]^{99},[-4]^{21}\right\}$ & $\{7,6,4,4 ; 1,1,1,6\}$ & Corol. 4 \\
\hline Biggs-Smith graph & $\begin{array}{l}\left\{[3]^{1},\left[\frac{1+\sqrt{17}}{2}\right]^{9},[2]^{18},\left[\theta_{1,2,3}\right]^{16,16,16},[0]^{17}\right. \\
\left.\quad\left[\frac{1-\sqrt{17}}{2}\right]^{9}\right\}\left(\theta_{1}, \theta_{2}, \theta_{3} \text { roots of } \theta^{3}+3 \theta^{2}\right. \\
\quad-3=0)\end{array}$ & $\begin{array}{l}\{3,2,2,2,1,1,1 ; \\
1,1,1,1,1,1,3\}\end{array}$ & Corol. 4 \\
\hline $\begin{array}{l}\text { Incidence graph } \\
\quad \operatorname{pg}(k-1, k-1, k-1) \\
\quad k=4,5,7,8\end{array}$ & $\left\{[k]^{1},[\sqrt{k}]^{k(k-1)},[0]^{2 k-2},[-\sqrt{k}]^{k(k-1)},[-k]^{1}\right\}$ & $\begin{array}{l}\{k, k-1, k-1,1 \\
\quad 1,1, k-1, k\}\end{array}$ & Corol. 5 \\
\hline $\begin{array}{l}\text { Coset graph doubly } \\
\text { truncated binary Golay }\end{array}$ & $\left\{[21]^{1},[5]^{210},[-3]^{280},[-11]^{21}\right\}$ & $\{21,20,16 ; 1,2,12\}$ & Corol. 6 \\
\hline $\begin{array}{l}\text { Coset graph extended } \\
\text { ternary Golay code }\end{array}$ & $\left\{[24]^{1},[6]^{264},[-3]^{440},[-12]^{24}\right\}$ & $\{24,22,20 ; 1,2,12\}$ & Prop. 1 \\
\hline
\end{tabular}

Table 2. Distance-regularity of graphs characterized by their spectra.

\begin{tabular}{lccc}
\hline Graph & Spectrum & Intersection array & Ref. \\
\hline $\begin{array}{c}\text { Collinearity graph } \\
\text { gen. octagon }\end{array}$ & $\left\{[s(t+1)]^{1},[s-1+\sqrt{2 s t}]^{m+},[s-1]^{m}\right.$, & $\{s(t+1), s t, s t, s t ; 1,1,1, t+1\}$ & Corol. 2 \\
$G O(s, t)$ & & & \\
Collinearity graph & $\left\{[2 s]^{1},[s-1 \pm \sqrt{3 s}]^{s(s+1)^{2}\left(s^{2}+s+1\right) / 6}\right.$, & $\{2 s, s, s, s, s, s ; 1,1,1,1,1,2\}$ & Corol. 3 \\
gen. dodecagon & {$[s-1 \pm \sqrt{s}]^{s(s+1)^{2}\left(s^{2}-s+1\right) / 2}$,} & & Corol. 5 \\
$G D(s, 1)$ & $\left.[s-1]^{2 s\left(s^{4}+s^{2}+1\right) / 3},[-2]^{s^{6}}\right\}$ & & \\
Incidence graph & $\left\{[s+1]^{1},[\sqrt{2 s+1-\alpha}]^{m},[0]^{v-2-2 m}\right.$, & $\{s+1, s, s, s+1-\alpha ;$ & $1,1, \alpha, s+1\}$ \\
partial geometry & $\left.[-\sqrt{2 s+1-\alpha}]^{m},[-s-1]^{1}\right\}$ & \\
$p g(s, s, \alpha)$ & $\left(v=2\left(1+s+\frac{s^{2}(s+1)}{\alpha}\right), m=\frac{s^{2}(s+1)^{2}}{\alpha(2 s+1-\alpha)}\right)$ & & \\
\hline
\end{tabular}

examples which were known to be determined by the corresponding intersection arrays, and therefore these are also determined by their spectra uniquely. For some graphs which seemed good candidates for being determined by their spectrum, we could prove that they do not satify the property by producing cospectral mates. These are given in Table 3 .

Additional motivation for the above questions comes from the following fundamental problem in the theory of graph spectra: Which graphs are determined by their spectrum? In general this is a very hard problem. Only for a small fraction of graphs the answer is known to be affirmative, yet it is conceivable that almost all graphs have the property. The difficulty is to prove the property for a given graph. Distance-regular graphs are an important source of examples for which this can be done. The present paper gives a few more examples. 
Table 3. Distance-regular graphs for which cospectral non-distance-regular graphs exist.

\begin{tabular}{llll}
\hline Graph & \multicolumn{1}{c}{ Spectrum } & \multicolumn{1}{c}{ Intersection array } & Ref. \\
\hline Wells graph & $\left\{[5]^{1},[\sqrt{5}]^{8},[1]^{10},[-\sqrt{5}]^{8},[-3]^{5}\right\}$ & $\{5,4,1,1 ; 1,1,4,5\}$ & Prop. 2 \\
Double Hoffman-Singleton & $\left\{[ \pm 7]^{1},[ \pm 3]^{21},[ \pm 2]^{28}\right\}$ & $\{7,6,6,1,1 ; 1,1,6,6,7\}$ & Prop. 3 \\
3-Cover $G Q(2,2)$ & $\left\{[6]^{1},[3]^{12},[1]^{9},[-2]^{18},[-3]^{5}\right\}$ & $\{6,4,2,1 ; 1,1,4,6\}$ & Prop. 4 \\
Foster graph & $\left\{[ \pm 3]^{1},[ \pm \sqrt{6}]^{12},[ \pm 2]^{9},[ \pm 1]^{18},[0]^{10}\right\}$ & $\{3,2,2,2,2,1,1,1 ;$ & Prop. 4 \\
& & $1,1,1,1,2,2,2,3\}$ & \\
\hline
\end{tabular}

\section{Vertex partitions}

Consider an arbitrary graph $G$ and let $\left\{X_{0}, \ldots, X_{m}\right\}$ be a partition of the vertex set $V$. Suppose each vertex from $X_{i}$ is adjacent to a constant number $x_{i, j}$, say, of vertices from $X_{j}$. Then we say that (the symbol) $x_{i, j}$ is well-defined. If $x_{i, j}$ is well-defined for all $i, j \in$ $\{0, \ldots, m\}$, then the vertex partition is called regular (or equitable) and the $(m+1) \times(m+1)$ matrix $\left(x_{i, j}\right)$ is called the quotient matrix. Given a vertex $x \in V$ with local diameter $d$, let $X_{i}=G_{i}(x)$ be the set of vertices at distance $i$ from $x$. Then $\left\{X_{0}, \ldots, X_{d}\right\}$ is called the distance partition with respect to $x$. Assume that, with respect to every vertex, the distance partition is regular with the same quotient matrix (which is clearly a tri-diagonal matrix), and that $G$ is connected. Then $G$ is distance-regular (by definition). The parameters of $G$ are $a_{i}=x_{i, i}, b_{i}=x_{i, i+1}, c_{i}=x_{i-1, i}, k_{i}=\left|X_{i}\right|$ and $k=k_{1}\left(i=0, \ldots, d\right.$; take $\left.b_{d}=c_{0}=0\right)$. They satisfy the following obvious conditions

$$
a_{i}+b_{i}+c_{i}=k, \quad k_{i-1} b_{i-1}=k_{i} c_{i}(i=1, \ldots, d), \quad k_{0}=c_{1}=1, \quad b_{0}=k_{1}=k .
$$

Thus all parameters of $G$ can be obtained from the intersection array $\Upsilon$.

For an arbitrary graph we say that $a_{i}$ is well-defined if for all distance partitions the $x_{i, i}$ 's are well-defined and mutually equal. For $b_{i}, c_{i}$ and $k_{i}$, well-defined is defined similarly. We shall call a multiset $\Sigma$ feasible for the spectrum of a distance-regular graph if there exists a feasible intersection array (according to the definition given in [1, p. 133]) that corresponds to $\Sigma$. In the following a somewhat more general definition of feasible is possible, but we will not go into the technical details.

\section{Distance-regular graphs with many unique geodesics}

Lemma 1 Let $G$ and $G^{\prime}$ be two graphs with the same spectrum, with $d+1$ distinct eigenvalues, and let $t \leq d$ be a positive integer. Suppose that in $G$ the parameters $a_{i}$, $b_{i}$, and $c_{i+1}=1, i=0, \ldots, t-1$ are well-defined. If in $G^{\prime}$ the parameters $a_{i}^{\prime}, b_{i}^{\prime}$, and $c_{i+1}^{\prime}$, $i=0, \ldots, t-2$ are well-defined, and the same as the corresponding intersection parameters of $G$, then also $a_{t-1}^{\prime}, b_{t-1}^{\prime}$, and $c_{t}^{\prime}$ are well-defined, and the same as in $G$.

Proof: First note that it immediately follows that for every vertex $x$ in $G$ or $G^{\prime}$ the number of vertices at distance $i, i=0, \ldots, t-1$ is determined by the given intersection parameters, and independent of $x$, say it is equal to $k_{i}, i=0, \ldots, t-1$. 
For $t=1$, the hypothesis says that $G$ is regular of valency $b_{0}$. Since one can see from the spectrum whether a graph is regular, and if so, its valency, this implies that $G^{\prime}$ is also regular with valency $b_{0}^{\prime}=b_{0}$. Note that $c_{1}^{\prime}$ is equal to one trivially.

Next, suppose that $t>1$. Let $A$ and $A^{\prime}$ be the adjacency matrices of $G$ and $G^{\prime}$, respectively. The arguments that we shall use are based on comparing $\operatorname{Tr}\left(A^{i}\right)=\operatorname{Tr}\left(A^{\prime i}\right)$ and the number of closed walks of length $i$ for $i=2 t-1$ and $i=2 t$, respectively.

For any vertex $x$ of $G$ or $G^{\prime}$, the number of closed walks of length $2 t-1$ starting (and ending) in $x$ is a constant plus the number of closed walks through $x$ where the points after steps $t-1$ and $t$ (halfway) are at distance $t-1$ from $x$. This follows since the number of walks besides the ones we explicitly mentioned can be expressed in terms of the known (and well-defined) intersection parameters. Thus it follows that the number of closed walks of length $2 t-1$ through $x$ in $G^{\prime}$ can be expressed as

$$
K+\sum_{y \in G_{t-1}^{\prime}(x)} a_{t-1}^{\prime}(x, y)
$$

where $a_{t-1}^{\prime}(x, y)$ is the number of neighbours of $y$ at distance $t-1$ from $x$ (for $y \in G_{t-1}^{\prime}(x)$ ), and $K$ is a constant which can be expressed in terms of the known intersection parameters. Since the number of closed walks of length $2 t-1$ through $x$ equals $\left(A^{\prime 2 t-1}\right)_{x x}$, it follows that

$$
\operatorname{Tr}\left(A^{\prime 2 t-1}\right)=v K+\sum_{x} \sum_{y \in G_{t-1}^{\prime}(x)} a_{t-1}^{\prime}(x, y)
$$

where $v$ denotes the number of vertices of $G^{\prime}$ (and $G$ ). Similarly one finds that

$$
\operatorname{Tr}\left(A^{2 t-1}\right)=v K+\sum_{x} \sum_{y \in G_{t-1}(x)} a_{t-1}
$$

and since $A$ and $A^{\prime}$ have the same spectrum, it follows that

$$
\sum_{x} \sum_{y \in G_{t-1}^{\prime}(x)} a_{t-1}^{\prime}(x, y)=\sum_{x} \sum_{y \in G_{t-1}(x)} a_{t-1}=v k_{t-1} a_{t-1} .
$$

Consequently, we find that if $c_{t}^{\prime}(x, y)$ is the number of neighbours of $y$ at distance $t-1$ from $x$ (for $y \in G_{t}^{\prime}(x)$ ), then by counting the number of edges between $G_{t-1}^{\prime}(x)$ and $G_{t}^{\prime}(x)$ in two ways it follows that

$$
\begin{aligned}
\sum_{x} \sum_{y \in G_{t}^{\prime}(x)} c_{t}^{\prime}(x, y) & =\sum_{x} \sum_{y \in G_{t-1}^{\prime}(x)}\left(k_{1}-c_{t-1}-a_{t-1}^{\prime}(x, y)\right) \\
& =v k_{t-1}\left(k_{1}-c_{t-1}-a_{t-1}\right)=v k_{t-1} b_{t-1} .
\end{aligned}
$$

Similarly, for any vertex $x$ of $G$ or $G^{\prime}$, the number of closed walks of length $2 t$ through $x$ is a constant plus the number of closed walks through $x$ where the points after steps $t-1, t$, and $t+1$ are at distance $t-1$ from $x$, plus the number of closed walks through $x$ where the point after step $t$ is at distance $t$, plus the number of closed walks through $x$ where the point 
after step $t$ is at distance $t-1$ from $x$ and so is exactly one of the points after steps $t-1$ and $t+1$. Thus the number of closed walks of length $2 t$ through $x$ in $G^{\prime}$ can be expressed as

$$
C+\sum_{y \in G_{t-1}^{\prime}(x)} a_{t-1}^{\prime}(x, y)^{2}+\sum_{y \in G_{t}^{\prime}(x)} c_{t}^{\prime}(x, y)^{2}+C^{\prime} \sum_{y \in G_{t-1}^{\prime}(x)} a_{t-1}^{\prime}(x, y),
$$

where $C$ and $C^{\prime}$ are constants which can be expressed in terms of the known intersection parameters. As before, it follows that

$$
\begin{aligned}
\operatorname{Tr}\left(A^{\prime 2 t}\right)= & v C+\sum_{x} \sum_{y \in G_{t-1}^{\prime}(x)} a_{t-1}^{\prime}(x, y)^{2}+\sum_{x} \sum_{y \in G_{t}^{\prime}(x)} c_{t}^{\prime}(x, y)^{2} \\
& +C^{\prime} \sum_{x} \sum_{y \in G_{t-1}^{\prime}(x)} a_{t-1}^{\prime}(x, y) .
\end{aligned}
$$

By Cauchy's inequality, and the fact that $c_{t}^{\prime}(x, y) \geq 1$, we obtain that

$$
\begin{aligned}
\operatorname{Tr}\left(A^{\prime 2 t}\right) \geq & v C+\frac{1}{v k_{t-1}}\left(\sum_{x} \sum_{y \in G_{t-1}^{\prime}(x)} a_{t-1}^{\prime}(x, y)\right)^{2} \\
& +\sum_{x} \sum_{y \in G_{t}^{\prime}(x)} c_{t}^{\prime}(x, y)+C^{\prime} \sum_{x} \sum_{y \in G_{t-1}^{\prime}(x)} a_{t-1}^{\prime}(x, y) \\
= & v C+\frac{1}{v k_{t-1}}\left(v k_{t-1} a_{t-1}\right)^{2}+v k_{t-1} b_{t-1}+C^{\prime} v k_{t-1} a_{t-1},
\end{aligned}
$$

with equality if and only if $a_{t-1}^{\prime}(x, y)$ is independent (and equal to $a_{t-1}$ ) of $x$ and $y$ ( $x$ and $y$ at mutual distance $t-1)$ and $c_{t}^{\prime}(x, y)=1$ for all $x$ and $y$ at mutual distance $t$.

The last expression is precisely $\operatorname{Tr}\left(A^{2 t}\right)$, which equals $\operatorname{Tr}\left(A^{\prime 2 t}\right)$, and so $a_{t-1}^{\prime}$ and $c_{t}^{\prime}$, and consequently $b_{t-1}^{\prime}$ are well-defined and equal to the corresponding parameters of $G$.

The actual existence of the graph $G$ in the assumptions of Lemma 1 is not necessary. All the necessary properties follow from the spectrum of $G^{\prime}$ and the well-defined intersection parameters; i.e., it follows that the equation

$$
\begin{aligned}
\operatorname{Tr}\left(A^{\prime 2 t}\right)= & v C+\frac{1}{v k_{t-1}}\left(\operatorname{Tr}\left(A^{\prime 2 t-1}\right)-v K\right)^{2}+v k_{t-1}\left(k_{1}-c_{t-1}\right) \\
& +\left(C^{\prime}-1\right)\left(\operatorname{Tr}\left(A^{\prime 2 t-1}\right)-v K\right)
\end{aligned}
$$

forces the parameters $a_{t-1}^{\prime}, b_{t-1}^{\prime}$, and $c_{t}^{\prime}$ to be well-defined.

Before applying Lemma 1 we mention another useful lemma; this lemma follows from the results in [6] (note specifically Lemma 5.1).

Lemma 2 If $G$ is a graph with spectrum $\Sigma$, which is feasible for a distance-regular graph and if the parameters $k_{i}, i=0,1, \ldots, d$, and $a_{i}, i=1, \ldots, d-2$ are well-defined, then $G$ is distance-regular. 
Somewhat weaker conditions than those in Lemma 2 are sufficient to prove distanceregularity, but for our purposes the made assumptions suffice. We could also use a deep theorem by Fiol and Garriga [3] which states that a graph is distance-regular if and only if for every vertex the number $k_{d}$ of vertices at distance $d$ (where $d+1$ is the number of distinct eigenvalues of the graph) equals an expression in terms of the spectrum. This theorem implies Lemma 2, but in the following we prefer not to refer to this result (for the sake of simplicity of our proofs), since we can avoid it without extra effort.

Theorem 1 If $G$ has the spectrum which is feasible for a distance-regular graph with diameter $d$ and $c_{i}=1$, for $i=1, \ldots, d-1$, then $G$ is such a distance-regular graph.

Proof: It follows by inductively applying Lemma 1 that all intersection parameters up to $c_{d-1}$ are well-defined, and hence that for every vertex the number of vertices $k_{i}$ at distance $i$, $i=0, \ldots, d$ is what is should be. It now follows from Lemma 2 that $G$ is distance-regular with the right parameters. (Instead of using Lemma 2 we could also use the counting arguments of the proof of Lemma 1: using Cauchy's inequality on the sum of $c_{d}^{\prime}(x, y)^{2}$ is helpful now, since at this stage we know the number of summands).

The case of diameter $d=3$ of Theorem 1 was already proven in [6]. Examples of distanceregular graphs with $c_{i}=1, i=1, \ldots, d-1$, are given by the (infinite family of) collinearity graphs of generalized polygons. For $d>2$ the intersection parameters of these graphs even determine that it is such a collinearity graph. For $d=2$ this is not true in general: for some generalized quadrangles there are so-called pseudo-geometric strongly regular graphs with the same parameters as the collinearity graph of the generalized quadrangle.

Corollary 1 Let $n>4$. If $G$ is a graph with the same spectrum as the collinearity graph of a generalized n-gon, then $G$ itself is such a graph.

This result for generalized polygons is new (as far as we know) for the generalized octagons $G O(s, t)$ and the generalized dodecagons $G D(s, 1)$. The spectra of these graphs can be obtained from [1, Section 6.5].

Corollary 2 If $G$ is a graph with spectrum $\left\{[s(t+1)]^{1},[s-1+\sqrt{2 s t}]^{m_{+}},[s-1]^{m}\right.$, $\left.[s-1-\sqrt{2 s t}]^{m_{-}},[-t-1]^{v-1-m_{+}-m-m_{-}}\right\}$, where $m_{ \pm}=\frac{1}{4}(t+1) \operatorname{stv} /\left(s(t-1)^{2}+t(s-1)^{2}+\right.$ $2 s t \pm(s-1)(t-1) \sqrt{2 s t}), m=\frac{1}{2}(t+1) s t v /\left(s(t-1)^{2}+t(s-1)^{2}+4 s t\right)$, and $v=$ $(1+s)(1+s t)\left(1+s^{2} t^{2}\right)$, then $G$ is the distance-regular collinearity graph of a generalized octagon $G O(s, t)$.

Corollary 3 If $G$ is a graph with spectrum $\left\{[2 s]^{1},[s-1 \pm \sqrt{3 s}]^{s(s+1)^{2}\left(s^{2}+s+1\right) / 6},[s-1 \pm\right.$ $\left.\sqrt{s}]^{s(s+1)^{2}\left(s^{2}-s+1\right) / 2},[s-1]^{2 s\left(s^{4}+s^{2}+1\right) / 3},[-2]^{s^{6}}\right\}$, then $G$ is the distance-regular collinearity graph of a generalized dodecagon $G D(s, 1)$.

Other examples of graphs satisfying the assumptions of Theorem 1 with $d>3$ are the Coxeter graph with intersection array $\{3,2,2,1 ; 1,1,1,2\}$ and spectrum $\left\{[3]^{1},[2]^{8},[-1+\right.$ $\left.\sqrt{2}]^{6},[-1]^{7},[-1-\sqrt{2}]^{6}\right\}$, the $M_{22}$ graph with intersection array $\{7,6,4,4 ; 1,1,1,6\}$ and 
spectrum $\left\{[7]^{1},[4]^{55},[1]^{154},[-3]^{99},[-4]^{21}\right\}$, and the Biggs-Smith graph with intersection array $\{3,2,2,2,1,1,1 ; 1,1,1,1,1,1,3\}$ and spectrum $\left\{[3]^{1},\left[\frac{1+\sqrt{17}}{2}\right]^{9},[2]^{18},\left[\theta_{1}\right]^{16},[0]^{17}\right.$, $\left.\left[\theta_{2}\right]^{16},\left[\frac{1-\sqrt{17}}{2}\right]^{9},\left[\theta_{3}\right]^{16}\right\}$, where $\theta_{1}, \theta_{2}$, and $\theta_{3}$ are the roots of the equation $\theta^{3}+3 \theta^{2}-3=0$. In fact, it follows from the uniqueness as a distance-regular graph (cf. [1, pp. 382, 368, 403]) that the last three graphs are all uniquely determined by their spectra. The same holds for (we restrict to the generalized polygons with $d>3$ here) the collinearity graphs of the generalized octagons $G O(s, 1)$ and $G O(1, s)$, for $s=2,3,4$, and the generalized dodecagons $G D(2,1)$ and $G D(1,2)$ (cf. [1, p. 204]).

Corollary 4 The Coxeter graph, the $M_{22}$ graph, the Biggs-Smith graph, and the collinearity graphs of the generalized octagons $G O(q, 1)$ and $G O(1, q), q=2,3,4$, and the generalized dodecagons $G D(2,1)$ and $G D(1,2)$ are uniquely determined by their spectra.

The uniqueness from the spectrum of the Coxeter graph was already proven in [6], by using its large girth (see Section 4). Also the results for the collinearity graphs of generalized polygons with line size 2 in Corollaries 1-4 follow from their large girths.

Fon-Der-Flaass [4] proved that there exists no distance-regular graph with intersection array $\{5,4,3,3 ; 1,1,1,2\}$. Thus Theorem 1 implies that there is no graph with spectrum $\left\{[5]^{1},[1+\sqrt{5}]^{32},[1]^{66},[1-\sqrt{5}]^{32},[-3]^{45}\right\}$.

\section{Distance-regular graphs with large girth}

In [2] (see also [6]) it was shown that a graph with the spectrum of a distance-regular graph with diameter $d$ and girth $g \geq 2 d-1$ is such a graph. For bipartite graphs this result can be improved.

Theorem 2 If $G$ has a spectrum which is feasible for a bipartite distance-regular graph with diameter $d$ and girth $g \geq 2 d-2$, then $G$ is such a distance-regular graph.

Proof: It follows from the bipartiteness (which is recognizable from the spectrum!), the girth (also recognizable from the spectrum), and Lemma 1 that the parameters $a_{0}=a_{1}=$ $\cdots=a_{d}=0, c_{1}=\cdots=c_{d-2}=1, b_{0}=k_{1}=k, b_{1}=\cdots=b_{d-2}=k-1$, and $k_{i}, i=$ $1, \ldots, d-2$ of $G$ are well-defined. Moreover, it now also follows from the bipartiteness of $G$ that the remaining parameters $k_{d-1}=\frac{1}{2} v-\sum_{i \neq 0} k_{d-1-2 i}$ and $k_{d}=\frac{1}{2} v-\sum_{i \neq 0} k_{d-2 i}$ are well-defined. But then it follows from Lemma 2 that $G$ is distance-regular.

Examples of bipartite distance-regular graphs with $g=2 d-2$ are the incidence graphs of partial geometries with equally many points and lines (cf. [1, p. 18]). For a partial geometry $p g(s, s, \alpha)$ the incidence graph is distance-regular with intersection array $\{s+1, s, s, s+$ $1-\alpha ; 1,1, \alpha, s+1\}(d=4, g=6)$.

More specifically, we have the incidence graphs of affine planes from which a parallel class of lines has been removed, i.e. a partial geometry $\operatorname{pg}(k-1, k-1, k-1)$, if $k$ is the order of the affine plane. Its incidence graph has intersection array $\{k, k-1, k-1,1 ; 1,1$, $k-1, k\}$ and spectrum $\left\{[k]^{1},[\sqrt{k}]^{k(k-1)},[0]^{2 k-2},[-\sqrt{k}]^{k(k-1)},[-k]^{1}\right\},($ cf. $[1$, p. 425$])$. It 
follows from the fact that the only affine planes of order $k$ less than 9 are Desarguesian, and that an affine plane is uniquely reconstructable from the $\operatorname{pg}(k-1, k-1, k-1)$, that the corresponding incidence graphs are uniquely determined by their spectra for $k=$ $2,3,4,5,7,8$. For $k=2$ this graph is the 8 -cycle, for $k=3$ it is the so-called Pappus graph.

Other examples are given by the incidence graph of the Van Lint-Schrijver partial geometry $p g(5,5,2)$ (cf. [1, p. 373]) which has intersection array $\{6,5,5,4 ; 1,1,2,6\}$ and spectrum $\left\{[6]^{1},[3]^{50},[0]^{60},[-3]^{50},[-6]^{1}\right\}$, and the incidence graphs of partial geometries $p g\left(n(n-1), n(n-1),(n-1)^{2}\right)$ related to Denniston arcs in projective planes $P G\left(2, n^{2}\right)$, where $n$ is a power of 2 . The latter graphs have intersection array $\left\{n^{2}-n+1, n(n-1)\right.$, $\left.n(n-1), n ; 1,1,(n-1)^{2}, n^{2}-n+1\right\}$ and spectrum $\left\{\left[n^{2}-n+1\right]^{1},[n]^{\left(n^{2}-n+1\right)^{2}},[0]^{2(n-1)\left(n^{2}+1\right)}\right.$, $\left.[-n]^{\left(n^{2}-n+1\right)^{2}},\left[-n^{2}+n-1\right]^{1}\right\}$ (cf. [1, p. 387]).

Corollary 5 If $G$ is a graph with spectrum $\left\{[s+1]^{1},[\sqrt{2 s+1-\alpha}]^{m},[0]^{v-2-2 m}\right.$, $\left.[-\sqrt{2 s+1-\alpha}]^{m},[-s-1]^{1}\right\}$, where $v=2\left(1+s+\frac{s^{2}(s+1)}{\alpha}\right)$ and $m=\frac{s^{2}(s+1)^{2}}{\alpha(2 s+1-\alpha)}$, then $G$ is the distance-regular incidence graph of a partial geometry $\operatorname{pg}(s, t, \alpha)$ with $t=s$. Moreover, if $s=\alpha=1,2,3,4,6,7$, and if $\alpha=1, s=2,3,4$, then $G$ is uniquely determined by its spectrum.

Note that if $\alpha=1$ the graphs are collinearity graphs of generalized octagons, which were already treated in the previous section.

In general, we cannot weaken the conditions on the girth and diameter for bipartite graphs: for example the Hoffman graph $[8]$ is a graph with the same spectrum $\left\{[4]^{1},[2]^{4},[0]^{6},[-2]^{4}\right.$, $\left.[-4]^{1}\right\}(d=4$ and $g=4)$ as the Hamming cube $H(4,2)$ (intersection array: $\{4,3,2,1 ; 1,2$, $3,4\}$ ), but it is not distance-regular. Also for the Desargues graph (intersection array: $\{3,2,2,1,1 ; 1,1,2,2,3\}$ ) there is a graph (cf. [7]) with the same spectrum, $\left\{[3]^{1},[2]^{4}\right.$, $\left.[1]^{5},[-1]^{5},[-2]^{4},[-3]^{1}\right\}(d=5$ and $g=6)$, but which is not distance-regular (see also Section 5).

For nonbipartite graphs we may need an extra condition:

Theorem 3 If $G$ has a spectrum $\Sigma$ which is feasible for a distance-regular graph with diameter $d$ and girth $g \geq 2 d-2$, and if the eigenvalues and the putative intersection parameters satisfy the condition $c_{d-1} c_{d}<-\left(c_{d-1}+1\right)\left(\lambda_{1}+\lambda_{2}+\ldots+\lambda_{d}\right)$ then $G$ is such a distance-regular graph.

Proof: Again, it follows from the girth and Lemma 1 that the parameters $a_{0}=a_{1}=\cdots=$ $a_{d-2}=0, c_{1}=\cdots=c_{d-2}=1, b_{0}=k_{1}=k, b_{1}=\cdots=b_{d-2}=k-1$, and $k_{i}, i=1, \ldots$, $d-2$ of $G$ are well-defined. An important role in this proof will be played by the Hoffmanpolynomial $h(x)=\prod_{i \neq 0}\left(x-\lambda_{i}\right)$. It is well-known (cf. [8]) that if $A$ is the adjacency matrix of $G$, then $h(A)=h(k) / v J$ (since $G$ is connected and regular), where $v$ is the number of vertices of $G$, and $J$ is the $v \times v$ all-ones matrix. From this equation it follows that if $x$ and $y$ are vertices at distance $d$, then $\left(A^{d}\right)_{x y}=h(k) / v$. However, in the putative distance-regular graph it should be the case that $\left(A^{d}\right)_{x y}=c_{d-1} c_{d}$, so we deduce that $h(k) / v=c_{d-1} c_{d}$.

If we now consider two vertices $x$ and $y$ at distance $d-1$, then the Hoffman-polynomial reveals that $\left(A^{d}\right)_{x y}=\left(\lambda_{1}+\cdots+\lambda_{d}\right)\left(A^{d-1}\right)_{x y}+c_{d-1} c_{d}$. This implies that $\left(A^{d-1}\right)_{x y} \leq$ 
$\frac{-c_{d-1} c_{d}}{\lambda_{1}+\cdots+\lambda_{d}}<c_{d-1}+1$ (the latter inequality follows from the extra condition), hence $c_{d-1}(x, y)$ $\leq c_{d-1}$, where $c_{d-1}(x, y)$ is the number of neighbours of $y$ at distance $d-2$ from $x$.

Since the parameter $a_{d-2}=0$ is well-defined, it also follows that for any vertex $x$ the number of edges between $G_{d-2}(x)$ and $G_{d-1}(x)$ is determined by the spectrum: with the earlier notation we have that

$$
\sum_{y \in G_{d-1}(x)} c_{d-1}(x, y)=k_{d-1} c_{d-1} .
$$

Moreover, by counting closed walks of length $2 d-2$ through $x$ (like we did in Lemma 1) we find that

$$
\sum_{x} \sum_{y \in G_{d-1}(x)} c_{d-1}(x, y)^{2}=v k_{d-1} c_{d-1}^{2}
$$

which is precisely what we want it to be. Combining the latter two equations and the inequality $c_{d-1}(x, y) \leq c_{d-1}$ gives us that

$$
v k_{d-1} c_{d-1}^{2}=\sum_{x} \sum_{y \in G_{d-1}(x)} c_{d-1}(x, y)^{2} \leq \sum_{x} \sum_{y \in G_{d-1}(x)} c_{d-1} c_{d-1}(x, y)=v k_{d-1} c_{d-1}^{2},
$$

hence the inequality is an equality. This means that the intersection parameter $c_{d-1}$ is welldefined, which implies that also the parameters $k_{d-1}$ and $k_{d}$ are. Like before, it now follows from Lemma 2 that $G$ is distance-regular.

Theorem 3 is in fact a generalization of Theorem 2: the extra condition in Theorem 3 is satisfied if the graph is bipartite, since in that case $c_{d}=k$ and $\lambda_{1}+\lambda_{2}+\cdots+\lambda_{d}=-k$.

It is unclear how tight the conditions of Theorem 3 are. There are many examples of distance-regular graphs with $g=2 d-3$ when $d=3$ (such as the Hamming graph $H(3,3)$ and the Johnson graphs $J(n, 3), n \geq 6$, cf. [6,7]) for which there are cospectral graphs which are not distance-regular; but we do not know of such examples with larger diameter. We also do not know if the extra condition on the intersection parameters and the eigenvalues is really necessary, but we expect it is (in general).

Examples of (non-bipartite) distance-regular graphs satisfying the assumptions of Theorem 3 are the folded 7 -cube with intersection array $\{7,6,5 ; 1,2,3\}$ and spectrum $\left\{[7]^{1},[3]^{21}\right.$, $\left.[-1]^{35},[-5]^{7}\right\}$, the coset graph of the doubly truncated binary Golay code with intersection array $\{21,20,16 ; 1,2,12\}$ and spectrum $\left\{[21]^{1},[5]^{210},[-3]^{280},[-11]^{21}\right\}$, the coset graph of the truncated binary Golay code with intersection array $\{22,21,20 ; 1,2,6\}$ and spectrum $\left\{[22]^{1},[6]^{330},[-2]^{616},[-10]^{77}\right\}$, and the coset graph of the binary Golay code with intersection array $\{23,22,21 ; 1,2,3\}$ and spectrum $\left\{[23]^{1},[7]^{506},[-1]^{1288},[-9]^{253}\right\}$. Note that the folded 7-cube and the coset graphs of the binary Golay code and the truncated binary Golay code are generalized Odd graphs, so for those graphs the result is not new (cf. [9]). Also, it follows that the folded 7-cube, and the coset graphs of the binary Golay code and the doubly truncated binary Golay code are uniquely determined by their respective spectra (since they are unique as distance-regular graphs, cf. [1, pp. 264, 361, 363]). 
Corollary 6 The folded 7-cube, the coset graph of the binary Golay code, and the coset graph of the doubly truncated binary Golay code are uniquely determined by their spectra. Moreover, any graph with the same spectrum as the coset graph of the truncated binary Golay code is distance-regular.

With similar techniques we can show the uniqueness from the spectrum of the unique distance-regular graph (cf. [1, p. 359]) with intersection array $\{24,22,20 ; 1,2,12\}$ : the coset graph of the extended ternary Golay code.

Proposition 1 The coset graph of the extended ternary Golay code is the unique graph with spectrum $\left\{[24]^{1},[6]^{264},[-3]^{440},[-12]^{24}\right\}$.

Proof: Let $G$ be a graph with the given spectrum. From the Hoffman-polynomial it follows that $A^{3}=-9 A^{2}+54 A+216 I+24 J$, where $A$ is the adjacency matrix of $G$. Like in the previous theorem, it follows that if $x$ and $y$ are two vertices at distance $2(=d-1)$, then $c_{2}(x, y) \leq 2$.

By counting the number of triangles in the graph (this number follows from the spectrum), it follows that $a_{1}(x, y)$, the number of common neighbours of two adjacent vertices $x$ and $y$, is $a_{1}=1$ on average. From the above cubic in $A$, it follows that $\left(A^{3}\right)_{x y}=78-9 a_{1}(x, y)$ for $x$ and $y$ adjacent. Now suppose that $a_{1}(x, y)=0$, then the number of walks of length 3 from $x$ to $y$ should be 78 . However, there can be at most 70, namely 23 walks of the form $x z x y(z \neq y ; z$ adjacent to $x), 23$ of the form $x y z y(z \neq x ; z$ adjacent to $y), 1$ of the form $x y x y$ and at most 23 of the form $x z w y$ ( $x$ adjacent to $z ; w$ adjacent to $z$ and $y ; z \neq y$ ). The latter follows from the fact that $w$ must be at distance 2 from $x$ and adjacent to $y$, and $w$ has at most two neighbours that are adjacent to $x$. So we have a contradiction, and it follows that the parameter $a_{1}=1$ is well-defined. It now follows by counting walks of length 4 through $x$, like in Theorem 3, that also $c_{2}=2$ is well-defined, and that $G$ is distance-regular.

\section{Switching in certain antipodal distance-regular graphs}

In this section we shall construct some graphs that are cospectral to certain antipodal distance-regular graphs, but which are not distance-regular themselves. These distanceregular graphs are in some sense close to satisfying the conditions of the above theorems. We use the following switching tool of Godsil and McKay [5].

Lemma 3 Let $G$ be a graph and let $\Pi=\left\{D, C_{1}, \ldots, C_{m}\right\}$ be a partition of the vertex set of $G$. Suppose that for every vertex $x \in D$ and every $i \in\{1, \ldots, m\}, x$ has either $0, \frac{1}{2}\left|C_{i}\right|$ or $\left|C_{i}\right|$ neighbours in $C_{i}$. Moreover, suppose that $\left\{C_{1}, \ldots, C_{m}\right\}$ is a regular partition of $G \backslash D$. Make a new graph $G^{\prime}$ as follows. For each $x \in D$ and $i \in\{1, \ldots, m\}$ such that $x$ has $\frac{1}{2}\left|C_{i}\right|$ neighbours in $C_{i}$ delete the corresponding $\frac{1}{2}\left|C_{i}\right|$ edges and join $x$ instead to the $\frac{1}{2}\left|C_{i}\right|$ other vertices in $C_{i}$. Then $G$ and $G^{\prime}$ have the same spectrum.

First we consider the Wells graph, the unique distance-regular graph with intersection array $\{5,4,1,1 ; 1,1,4,5\}$ : a double cover of the complement of the Clebsch graph (cf. [1, p. 
266]). Its spectrum is $\left\{[5]^{1},[\sqrt{5}]^{8},[1]^{10},[-\sqrt{5}]^{8},[-3]^{5}\right\}$. In this graph we take an edge $\{x, y\}$ and its antipodal edge $\left\{x^{\prime}, y^{\prime}\right\}$ (where $x^{\prime}$ and $y^{\prime}$ are the antipodal vertices of $x$ and $y$, respectively). A new graph is constructed by removing these two edges, and connecting $x$ to $x^{\prime}$ and $y$ to $y^{\prime}$ (i.e. two edges are switched into two new edges). Then we have:

Proposition 2 The graph obtained from the Wells graph by the above switching operation has the same spectrum as the Wells graph, and it is not distance-regular.

Proof: Write $\Gamma_{z}$ for the set of neighbours of the vertex $z$ and consider the following vertex partition of the Wells graph.

$$
\left\{\{x\},\{y\},\left\{x^{\prime}\right\},\left\{y^{\prime}\right\}, \Gamma_{x} \backslash\{y\}, \Gamma_{y} \backslash\{x\}, \Gamma_{x^{\prime}} \backslash\left\{y^{\prime}\right\}, \Gamma_{y^{\prime}} \backslash\left\{x^{\prime}\right\}, \Delta\right\},
$$

where $\Delta$ consists of the remaining vertices. It follows from the parameters of the Wells graph that the partition is regular with quotient matrix

$\left[\begin{array}{llll|l|ll|l|l}0 & 1 & 0 & 0 & 4 & 0 & 0 & 0 & 0 \\ 1 & 0 & 0 & 0 & 0 & 4 & 0 & 0 & 0 \\ 0 & 0 & 0 & 1 & 0 & 0 & 4 & 0 & 0 \\ 0 & 0 & 1 & 0 & 0 & 0 & 0 & 4 & 0 \\ \hline 1 & 0 & 0 & 0 & 0 & 0 & 0 & 1 & 3 \\ \hline 0 & 1 & 0 & 0 & 0 & 0 & 1 & 0 & 3 \\ 0 & 0 & 1 & 0 & 0 & 1 & 0 & 0 & 3 \\ \hline 0 & 0 & 0 & 1 & 1 & 0 & 0 & 0 & 3 \\ \hline 0 & 0 & 0 & 0 & 1 & 1 & 1 & 1 & 1\end{array}\right]$.

Next we make a new partition $\left\{D, C_{1} \ldots, C_{4}\right\}$ by uniting classes as indicated by the lines in the above matrix. That is,

$$
\begin{aligned}
D & =\left\{x, y, x^{\prime}, y^{\prime}\right\}, \quad C_{1}=\Gamma_{x} \backslash\{y\}, \quad C_{2}=\left(\Gamma_{y} \cup \Gamma_{x^{\prime}}\right) \backslash\left\{y^{\prime}, x\right\}, \\
C_{3} & =\Gamma_{y^{\prime}} \backslash\left\{x^{\prime}\right\}, \quad \text { and } \quad C_{4}=\Delta .
\end{aligned}
$$

It follows directly from the above quotient matrix that this new partition satisfies the hypothesis of the above lemma, and the switching operation described above produces the cospectral graph of the lemma (interchange $x^{\prime}$ and $y$ ).

To show that the new graph is not distance-regular, consider a vertex $z \in \Gamma_{x} \backslash\{y\}$ and its antipode $z^{\prime}$. It is easy to see that in the new graph there is a unique path of length three between $z$ and $z^{\prime}$. This suffices to show that the new graph is not distance-regular, since otherwise there would have been $c_{3}=4$ such paths.

In fact, E. Spence (private communication) has found exactly three graphs with the spectrum of the Wells graph by an exhaustive computer search. The third graph can be 
obtained from the second one (the one just constructed) by switching (in the same way) two antipodal edges in $\Delta$, the set of vertices at distance two from the vertices $x, y, x^{\prime}$, and $y^{\prime}$.

In the same way as for the Wells graph, a cospectral graph for the bipartite double (cover) of a Moore graph of diameter two can be obtained by switching. Such a bipartite double has intersection array $\{k, k-1, k-1,1,1 ; 1,1, k-1, k-1, k\}$. It is well-known that unique examples exist for $k=2,3,7$ (the bipartite doubles of the 5-cycle, the Petersen graph, and the Hoffman-Singleton graph), and that the only other possible case $k=57$ is still open (cf. [1, p. 207]). The proof that the switched graphs have the same spectrum as the original graphs is like in the previous proposition: here the starting partition has ten cells: the last cell $\Delta$ is split into two parts according to the bipart to which the vertices belong.

A similar argument as before shows that the new graphs are not distance-regular, except for $k=2$, where the "new" graph is still a 10-cycle. The switched example for the Desargues graph (the bipartite double of the Petersen graph) is the same as the one obtained in [7], although the construction method looks different at first sight.

Proposition 3 The bipartite double of the Petersen graph, the bipartite double of the Hoffman-Singleton graph, and the bipartite double of a putative Moore graph with valency 57 are not uniquely determined by their spectra.

Also the unique distance-regular graph with intersection array $\{6,4,2,1 ; 1,1,4,6\}$ : an antipodal triple cover of $G Q(2,2)$ (cf. [1, p. 398]) can be switched into a graph with the same spectrum, and which is not distance-regular.

Here we take a triangle, and its two antipodal triangles. The switching consists of removing the edges in the three triangles and connecting the antipodes. That is, the three triangles $\{x, y, z\},\left\{x^{\prime}, y^{\prime}, z^{\prime}\right\}$ and $\left\{x^{\prime \prime}, y^{\prime \prime}, z^{\prime \prime}\right\}$ are switched into three new triangles $\left\{x, x^{\prime}, x^{\prime \prime}\right\}$, $\left\{y, y^{\prime}, y^{\prime \prime}\right\}$ and $\left\{z, z^{\prime}, z^{\prime \prime}\right\}$. Here we apply Lemma 3 with the partition $\left\{D, C_{1}, \ldots, C_{6}\right\}$, where

$$
\begin{aligned}
D & =\left\{x, y, z, x^{\prime}, y^{\prime}, z^{\prime}, x^{\prime \prime}, y^{\prime \prime}, z^{\prime \prime}\right\}, \quad C_{1}=\Gamma_{x} \backslash D, \quad C_{2}=\Gamma_{y^{\prime}} \backslash D, \quad C_{3}=\Gamma_{z^{\prime \prime}} \backslash D, \\
C_{4} & =\left(\Gamma_{y} \cup \Gamma_{x^{\prime}}\right) \backslash D, \quad C_{5}=\left(\Gamma_{z} \cup \Gamma_{x^{\prime \prime}}\right) \backslash D, \quad C_{6}=\left(\Gamma_{z^{\prime}} \cup \Gamma_{y^{\prime \prime}}\right) \backslash D .
\end{aligned}
$$

It is straightforward to check that this partition indeed satisfies the hypothesis of Lemma 3 , and that the cospectral graph obtained by the lemma is the one described above. The new graph is not distance-regular by a similar argument as before.

There is another interesting graph related to the triple cover of $G Q(2,2)$ : the Foster graph. This graph is the incidence graph of the partial linear space of vertices (points) and triangles (lines) in the triple cover; and it is the unique distance-regular graph with intersection array $\{3,2,2,2,2,1,1,1 ; 1,1,1,1,2,2,2,3\}$ (cf. [1, p. 398]; geometers call the partial linear space the tilde-geometry). The switching operation we applied to the triple cover of $G Q(2,2)$ is essentially a switching operation in the corresponding partial linear space (three lines are switched into three new lines), and from this it will follow that also the Foster graph has a cospectral graph that is not distance-regular.

Proposition 4 The distance-regular triple cover of $G Q(2,2)$ and the Foster graph are not uniquely determined by their spectra. 
Proof: For the triple cover of $G Q(2,2)$ this follows from the above arguments.

For the Foster graph the result follows from the observation that there is a one-one relation between graphs with spectrum $\left\{[6]^{1},[3]^{12},[1]^{9},[-2]^{18},[-3]^{5}\right\}$ (that of the cover of $G Q(2,2))$ and graphs with spectrum $\left\{[ \pm 3]^{1},[ \pm \sqrt{6}]^{12},[ \pm 2]^{9},[ \pm 1]^{18},[0]^{10}\right\}$ (that of the Foster graph). To show this relation, we first consider any graph with the first spectrum. The distance-regular graph with this spectrum has parameters $k_{1}=6, a_{1}=1$, and $c_{2}=1$, and so it follows from Lemma 1 that also the graph under consideration has these well-defined parameters. This implies among others that every edge in the graph is in a unique triangle. So if we call vertices points and triangles lines, then this defines a partial linear space. If $N$ is the incidence matrix of this partial linear space, then the adjacency matrix of the collinearity graph is given by $N N^{T}-3 I$ and the adjacency matrix of the incidence graph of the partial linear space is given by

$$
\left[\begin{array}{cc}
O & N \\
N^{T} & O
\end{array}\right]
$$

and it easily follows that the spectrum of the incidence graph is the same as the spectrum of the Foster graph.

Conversely, consider a graph with the same spectrum as the Foster graph. Since the Foster graph is bipartite distance-regular with parameters $k_{1}=3$ and $c_{2}=1$, it follows that also the graph under consideration is bipartite with these well-defined parameters. This implies that if the matrix $N$ describes the adjacencies between the two biparts in the graph, then $N N^{T}-3 I$ is the adjacency matrix of a graph (one of the so-called halved graphs), which has the same spectrum as the triple cover of $G Q(2,2)$.

So there is a one-one correspondence between graphs with the earlier mentioned spectra.

\section{Appendix}

In this appendix we sketch a proof of the following result: for a distance-regular graph the spectrum determines the intersection array. This less-known but relevant result (mentioned in the introduction) has been observed before, but it doesn't seem to be readily available in the literature.

Lemma 4 If $G$ is a distance-regular graph, then the intersection array $\Upsilon$ is determined by the spectrum $\Sigma$.

Proof: Let $G$ be a distance-regular graph with spectrum $\Sigma=\left\{\left[\lambda_{0}\right]^{m_{0}},\left[\lambda_{1}\right]^{m_{1}}, \ldots,\left[\lambda_{d}\right]^{m_{d}}\right\}$, where $\lambda_{0}$ is the largest eigenvalue. The distance polynomial $p_{i}$ of degree $i$ satisfies the equation $A_{i}=p_{i}(A)$, where $A_{i}$ is the distance- $i$ adjacency matrix of $G, i=0, \ldots, d$, and $A=A_{1}$. The orthogonality relations of the underlying association scheme of $G$ state that

$$
\sum_{i=0}^{d} m_{i} p_{h}\left(\lambda_{i}\right) p_{j}\left(\lambda_{i}\right)=v p_{j}\left(\lambda_{0}\right) \delta_{h j}
$$


It now follows (by induction on the degree $i$ with $p_{0}(x)=1$; Gram-Schmidt orthogonalisation) that these relations uniquely determine the distance polynomials of $G$ from $\Sigma$. From the distance polynomials all intersection parameters follow.

\section{Acknowledgment}

We thank Ted Spence for determining all (three) graphs with the spectrum of the Wells graph.

\section{References}

1. A.E. Brouwer, A.M. Cohen, and A. Neumaier, Distance-Regular Graphs, Springer, Heidelberg, 1989.

2. A.E. Brouwer and W.H. Haemers, "The Gewirtz graph: An exercise in the theory of graph spectra," European J. Combin. 14 (1993), 397-407.

3. M.A. Fiol and E. Garriga, "From local adjacency polynomials to locally pseudo-distance-regular graphs," J. Comb. Th. B. 71 (1997), 162-183.

4. D.G. Fon-Der-Flaass, "A distance-regular graph with intersection array $\{5,4,3,3 ; 1,1,1,2\}$ does not exist," J. Alg. Comb. 2 (1993), 49-56.

5. C.D. Godsil and B.D. McKay, "Constructing cospectral graphs," Aequationes Mathematicae 25 (1982), 257_ 268.

6. W.H. Haemers, "Distance-regularity and the spectrum of graphs," Linear Alg. Appl. 236 (1996), 265-278.

7. W.H. Haemers and E. Spence, "Graphs cospectral with distance-regular graphs," Linear Multilin. Alg. 39 (1995), 91-107.

8. A.J. Hoffman, "On the polynomial of a graph," Amer. Math. Monthly 70 (1963), 30-36.

9. T. Huang and C. Liu, "Spectral characterization of some generalized odd graphs," Graphs Comb. 15 (1999), 195-209. 